\title{
Scalable Synthesis of Acridinium Catalysts for Photoredox Deuterations
}

\section{Bouthayna Zilate \\ Christian Fischer \\ Lukas Schneider \\ Christof Sparr*}

Department of Chemistry, University of Base St. Johanns-Ring 19, 4056 Basel, Switzerland christof.sparr@unibas.ch

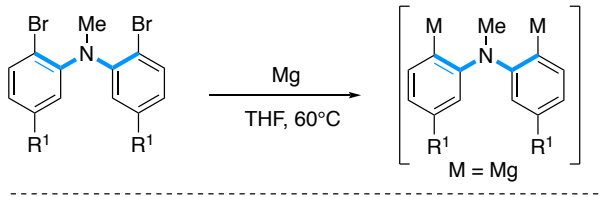<smiles>[R7]c1ccc([R])c2cc([Tl])ccc12</smiles><smiles>C[N+](C)(Cl)CCCN1c2ccccc2CCc2ccc(Cl)cc21</smiles>

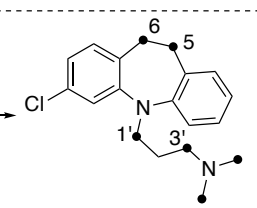

Abstract The continuous development of photocatalytic methods incentivizes the design of organic catalysts to complement the frequently used and precious polypyridyl transition metal systems. Herein, we describe a scalable synthesis of suitable acridinium dyes and their application in photoredox deuterations. The acridinium catalysts, prepared in multi-gram scale, allowed the deuteration of a pharmaceutically relevant scaffold in high yield and selectivity under mild conditions.

Key words acridinium salts, deuteration, Grignard reactions, photoredox catalysis, scalability

Acridinium salts, pioneered by Fukuzumi ${ }^{1}$ and utilized for various innovative methodologies by Nicewicz, ${ }^{2}$ are established as particularly valuable catalysts for photoredox chemistry (Scheme 1). As organocatalysts, efficient and scalable processes for their synthesis would render them potentially applicable for industrial applications. To further expand their accessibility, we thus developed a method for the preparation of acridinium salts by a twofold addition of a 1,5-bifunctional organometallic reagent to an ester ${ }^{3}$ while Nicewicz and co-workers recently disclosed an alternative synthesis of acridinium salts based on late stage nucleophilic substitutions of xanthilium dyes. ${ }^{4}$ Since the photostability of mesityl acridinium catalysts is impacted by the oxidation of the para-methyl group, ${ }^{5}$ methods allowing variation of this moiety are particularly valuable. Owing to the expedient accessibility of 1,5-bifunctional organomagnesium reagents, 6 we anticipated that acridinium salts with different redox properties can be readily prepared on gram scale. Furthermore, our direct acridinium synthesis from esters allows to incorporate electron-donating groups such as amino functionalities, that impactfully modulate the properties of the organophotoredox catalysts. We hence set out to explore the synthesis of acridinium salts with and without amino groups, their scale-up and the performance in a photoredox deuteration as benchmark reaction.

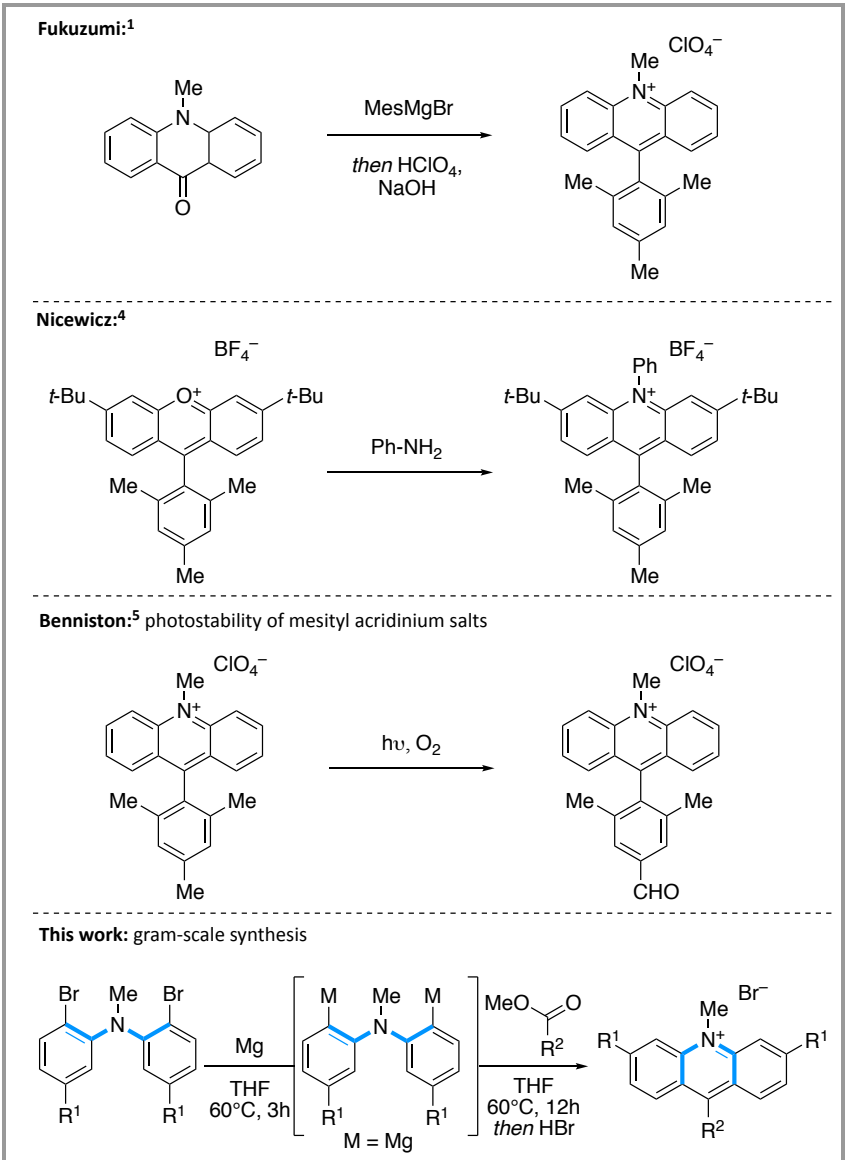

Scheme 1 Syntheses and photostability of acridinium photocatalysts 
Our synthesis of the acridinium catalysts $\mathbf{1}$ started with the dibromophenyl- $N$-methylaniline precursor $\mathbf{2 a}$, which was obtained through a Buchwald-Hartwig amination followed by a methylation (Scheme 2). ${ }^{7}$ The formation of the 1,5-bifunctional organometallic reagent, nucleophilic addition to ester substrates and dehydration using $\mathrm{HBr}^{3 a}$ led to acridinium salts $\mathbf{1 a}$ and $\mathbf{1 b}$ in good yields (63\% and 78\%, Scheme 2 ). Encouraged by these results and the distinctive catalytic performance of organophotocatalyst 1c ${ }^{\text {3a }}$ we next tackled the gram-scale synthesis of $\mathbf{1 c}$ and $\mathbf{1 d}$. The former preparation of reagents $\mathbf{3}$ and 4 for the $\mathrm{C}-\mathrm{N}$ cross coupling proved challenging during scale-up and led us to circumvent the Sandmeyer iodination from 3 to $4 .^{8}$ Gratifyingly, an alternative route consisting of a reductive amination, a Finkelstein reaction and bromination ${ }^{9}$ allowed us to access intermediate $\mathbf{4}$ in good to excellent yields without any column chromatography. A careful optimization of the nitration and reduction conditions delivered intermediate 3 in good yields, purified by recrystallisation. ${ }^{10}$ With the two coupling partners in hand, a C-N cross-coupling, followed by a methylation were performed. ${ }^{7}$ After recrystallisation, we received $35 \mathrm{~g}$ of the key intermediate $\mathbf{2 b}$. Similarly, the formation of the 1,5-bifunctional organometallic reagent was achieved on large scale, using elemental magnesium in refluxing THF. Addition of the different carboxylic acid esters to the reagents and $\mathrm{HBr}$ treatment provided $3.9 \mathrm{~g}$ of $\mathbf{1 c}$ and $\mathbf{1 d}$ in $83 \%$ and $84 \%$ yield, respectively.

Interestingly, catalyst 1a exhibited a higher excited state reduction potential (Table $1, \mathrm{E}_{1 / 2}\left[\mathrm{P}^{*} / \mathrm{P}^{-}\right]=+2.32 \mathrm{~V}$ vs $\mathrm{SCE}$ ) compared to the Fukuzumi catalyst $\left(\mathrm{E}_{1 / 2}\left[\mathrm{P}^{*} / \mathrm{P}^{-}\right]=+2.18 \mathrm{~V}\right.$ vs SCE). ${ }^{1}$ On the other hand, $\mathbf{1 b}$ displayed a similar excited state reduction potential $\left(\mathrm{E}_{1 / 2}\left[\mathrm{P}^{*} / \mathrm{P}^{-}\right]=+2.21 \mathrm{~V}\right.$ vs $\left.\mathrm{SCE}\right)$, indicating the influence of electron density at the phenyl moiety. The catalyst 1d without the labile para-methyl group showed almost identical properties as compared to $1 \mathrm{c}\left(\mathrm{E}_{1 / 2}\left[\mathrm{P}^{*} / \mathrm{P}^{-}\right]=+1.25 \mathrm{~V}\right.$ vs SCE) which makes it an ideal candidate for further investigations in photoredox catalysis.

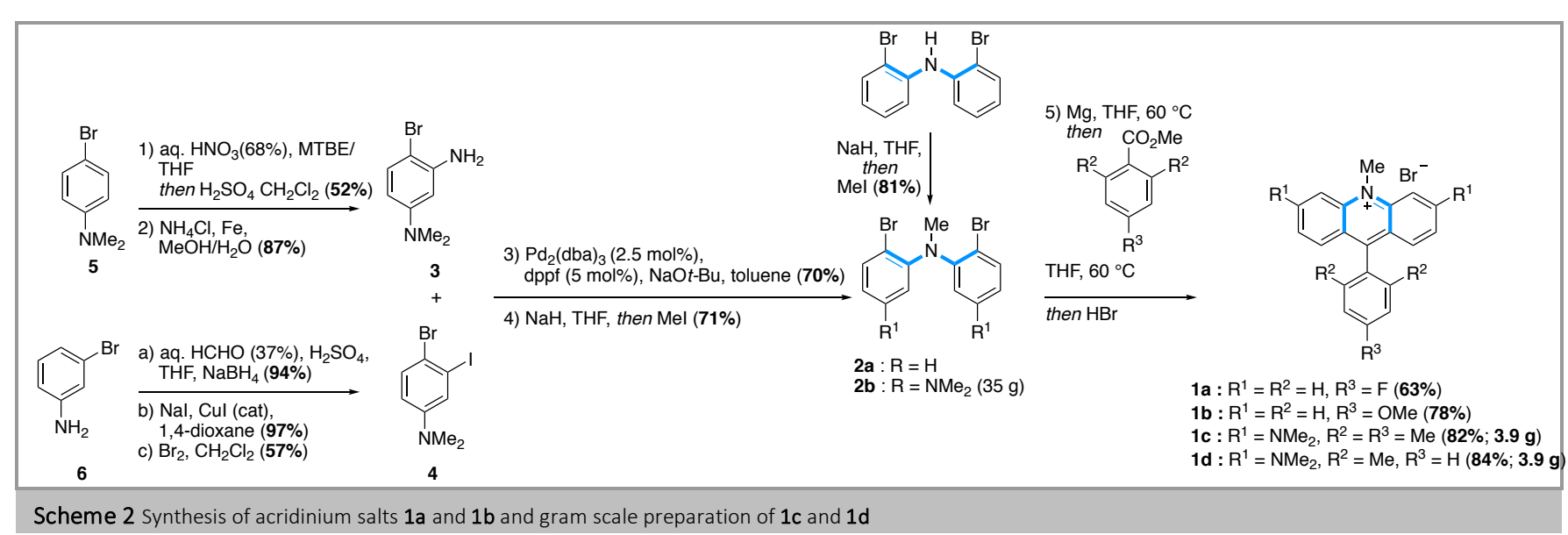

Scheme 2 Synthesis of acridinium salts $1 \mathrm{a}$ and $1 \mathrm{~b}$ and gram scale preparation of $1 \mathrm{c}$ and $1 \mathrm{~d}$
Table 1 Photophysical properties of the acridinium photocatalysts<smiles></smiles><smiles>Cc1cc([N+](=O)[O-])cc(C)c1Cc1c2ccc(N(C)[N+](=O)[O-])cc2[n+]([O-])c2cc([N+](=O)[O-])ccc12</smiles>

$1 c$

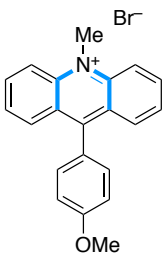

1b

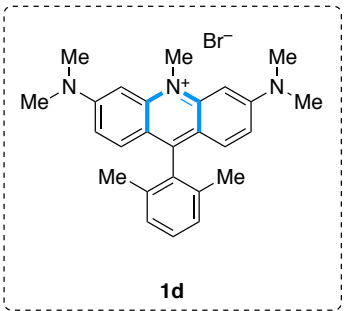

\begin{tabular}{cccccc}
\hline Dye & $\begin{array}{c}\lambda_{\text {abs }} \\
{[\mathrm{nm}]^{\mathrm{a}}}\end{array}$ & $\begin{array}{c}\lambda_{\text {em }} \\
{[\mathrm{nm}]^{\mathrm{a}}}\end{array}$ & $\begin{array}{c}E_{0,0} \\
{[\mathrm{eV}]^{\mathrm{a}}}\end{array}$ & $\begin{array}{c}E_{1 / 2}\left(\mathrm{P} / \mathrm{P}^{-}\right) \\
{[\mathrm{V}]^{\mathrm{b}}}\end{array}$ & $\begin{array}{c}E_{1 / 2}\left(\mathrm{P}^{*} / \mathrm{P}^{-}\right) \\
{[\mathrm{V}]}\end{array}$ \\
\hline 1a & 426 & 512 & 2.83 & -0.51 & +2.32 \\
1b & 438 & 499 & 2.77 & -0.56 & +2.21 \\
1c & 503 & 530 & 2.40 & -1.15 & +1.25 \\
1d & 504 & 533 & 2.40 & -1.15 & +1.25 \\
\hline
\end{tabular}

a Measured in MeCN $\left(15 \mu \mathrm{molL}^{-1}\right)$.

${ }^{b}$ Measured in 0.1 molL $^{-1} \mathrm{n}-\mathrm{Bu}_{4} \mathrm{~N} \cdot \mathrm{PF}_{6}$ in dry, degassed MeCN against SCE.

To explore the catalytic performance of the acridinium catalysts, we studied the deuteration of clomipramine as benchmarking reaction. ${ }^{11}$ An average isotopic incorporation of 7 deuterium atoms per molecule with a distribution between aliphatic and benzylic positions ( $\left.\mathrm{C} 5, \mathrm{C} 6, \mathrm{C1}^{\prime}, \mathrm{C}^{\prime}, \mathrm{NMe}_{2}\right)$ has been reported by MacMillan and coworkers using 4CzIPN as photocatalyst. We thus tested several acridinium photocatalysts, ${ }^{12}$ under identical reaction conditions (Table 2). Gratifyingly, a high selectivity for the aliphatic positions ( $\mathrm{C1}^{\prime}=20 \%, \mathrm{C}^{\prime}=64 \%, \mathrm{NMe}_{2}=39 \%$ ), with an average of 4 deuterium atoms per molecule was observed when using $1 \mathbf{c}$ with $1 \mathrm{~mol} \%$ catalyst loading. A similar pattern was observed when employing 1d with an even higher selectivity for the C3' position (70\%) and an identical average incorporation of 4 deuterium atoms per molecule. 


(1.0)

a Reaction performed with clomipramine $\mathrm{HCl}(100 \mu \mathrm{mol}), \mathrm{Li}_{2} \mathrm{CO}_{3}(480 \mu \mathrm{mol})$, triisopropylsilanethiol (30 mol\%), photocat. (2.5 mol\%) unless stated otherwise, $\mathrm{D}_{2} \mathrm{O}$ $(5.00 \mathrm{mmol})$ in NMP $(1.6 \mathrm{~mL})$ at RT, $24 \mathrm{~h}$ irradiation with Kessil A160WE tuna blue.

b Photocat. (1 mol\%).

$c$ Yield of isolated products.

${ }^{d}$ Determined by ${ }^{1} \mathrm{H}-\mathrm{NMR}$ of the free base.

In conclusion, we describe an efficient synthesis to access acridinium salts with suitable photophysical properties. The direct transformation of esters into acridinium catalysts allowed the large-scale synthesis of amino-functionalized photocatalysts with attenuated photoredox properties. The utility of the catalysts was demonstrated by the deuteration of clomipramine, providing high selectivity for aliphatic positions using low catalyst loadings.

All reactions were carried out in dried glassware under an $\mathrm{Ar}$ atmosphere. THF (99.5\%, Extra Dry, over Molecular Sieves, Stabilized, AcroSeal ${ }_{\text {, }}$ Code: 348455000) was purchased from Acros Organics. All starting materials and reaction solvents were purchased from commercial sources and used without further purification. Unless noted otherwise, all photoreactions were performed in a sealed Biotage $(2-5 \mathrm{~mL}$ microwave vial equipped with a $10 \mathrm{~mm} \times 5 \mathrm{~mm}$ magnetic stir bar stirring at $1400 \mathrm{rpm}$. The vial was placed on a stirring plate laterally in $3 \mathrm{~cm}$ distance to a Kessil LED A160WE Tuna Blue, $40 \mathrm{~W}$, adjusted to maximum intensity and white ( $\lambda$ max: $464 \mathrm{~nm}$ ). A sideward fan was used to keep ambient temperature $\left(\sim 30^{\circ} \mathrm{C}\right)$. All starting materials and reaction solvents were purchased from commercial sources and used without further purification. Solvents for extractions and chromatography were technical grade and distilled prior to use. Analytical thin layer chromatography (TLC) was performed on pre-coated Merck silica gel $60 \mathrm{~F}_{254}$ plates $(0.25 \mathrm{~mm})$ and visualised by UV. Flash column chromatography was carried out on Silicycle SiliaFlash P60 (230-400 mesh). Concentration in vacuo was performed by rotary evaporation to $\sim 10 \mathrm{mbar}$ at $40{ }^{\circ} \mathrm{C}$ unless stated otherwise. ${ }^{1} \mathrm{H}$ NMR and ${ }^{13} \mathrm{C}$ NMR spectra were recorded on a Bruker Avance III $500 \mathrm{MHz}$ spectrometer at $298 \mathrm{~K} \mathrm{in} \mathrm{CDCl}_{3}$ or MeOD supplied by Cambridge Isotope Laboratories (DLM-7TB-100S). Chemical shifts $(\delta)$ are reported in ppm relative to tetramethylsilane $(0.00 \mathrm{ppm})$. The multiplicities are reported in $\mathrm{Hz}$ as: $\mathrm{s}=$ singlet, $\mathrm{br}=$ broad singlet, $\mathrm{d}=$ doublet, $\mathrm{t}=$ triplet, $\mathrm{q}=$ quartet and $\mathrm{m}=$ multiplet. Melting points were measured on a Büchi $M-565$ melting point apparatus and are uncorrected. IR spectra were measured on an ATR Varian Scimitar 800 FT-IR spectrometer and reported in $\mathrm{cm}^{-1}$. The intensities of the bands are reported as: $\mathrm{w}=$ weak, $\mathrm{m}=$ medium, $\mathrm{s}=$ strong. High-resolution mass spectrometry (HR-ESI) was performed by Dr. Michael Pfeffer of the University of Basel on a Bruker maXis 4G QTOF ESI mass spectrometer. Cyclic Voltammetry was performed in dry, degassed $0.1 \mathrm{molL}^{-1}$ tetra nbutylammonium hexafluorophosphate in MeCN. Voltammograms were recorded with a Versastat3-200 potentiostat from Princeton Applied Research employing a glassy carbon disk working electrode, SCE reference electrode and a silver wire counter electrode and a potential sweep rate of $0.1 \mathrm{Vs}^{-1}$. The glassy carbon electrode and Ag wire were polished prior to measurement. UV/Vis spectroscopy was performed on a Shimadzu UV-1650 PC spectrometer in acetonitrile using Hellma fluorescence cells (111-QS, light path: $10 \mathrm{~mm}$ ). Molar absorption coefficients $(\varepsilon)$ were determined at the wavelength of maximum absorbance (labs) of a $\sim 10 \mu \mathrm{molL}^{-1}$ dye solution. Steady-state emission spectroscopy was carried out with a Fluorolog-3-22 instrument from Horiba Jobin-Yvon. Excitation occurred at the long-wavelength absorption band of the respective dye. All emission spectra were recorded in argon-saturated acetonitrile, and strongly diluted dye solutions (c $<15$ $\mu \mathrm{mol} \mathrm{L}-1$ ) were used to avoid inner filter effects. All spectra so obtained were corrected for the wavelength dependent sensitivity of the spectrometer. Additional emission spectra of the pure solvent at all excitation and detection conditions were measured to ensure the absence of stray light or impurity signals.

Synthesis of Acridinium Salts $\mathbf{1 a}$ and $\mathbf{1 b}$

\section{2-Bromo- $N$-(2-bromophenyl)- $N$-methylaniline}

\section{[CAS Reg. No.:87345-09-3]}

Prepared according to a modified literature procedure. ${ }^{7,13}$ Bis-(2-bromophenyl)amine (4.59 g, $14.0 \mathrm{mmol})$ in THF $(70 \mathrm{~mL})$ was treated with sodium hydride $(60 \%$ dispersion in mineral oil, $700 \mathrm{mg}, 17.5$ $\mathrm{mmol}$ ) at RT and refluxed for $30 \mathrm{~min}$. Iodomethane (3.78 g, $1.66 \mathrm{~mL}, 26.6$ mmol) was added and the mixture was refluxed for another two hours, cooled to RT and treated with $\mathrm{H}_{2} \mathrm{O}(50 \mathrm{~mL})$. The resulting mixture was extracted with $\mathrm{Et}_{2} \mathrm{O}$ ( $3 \times 65 \mathrm{~mL}$ ), the combined organic layers were dried over $\mathrm{Na}_{2} \mathrm{SO}_{4}$ and concentrated in vacuo. The residue was washed with pentane and dried in vacuo to obtain a white solid (3.87 g, 81\%, m.p. 106$\left.108^{\circ} \mathrm{C}\right)$.

IR (ATR, neat): 3434w, 3286w, 2959w, 2872w, 166w, 1544w, 1468m, $1341 \mathrm{w}, 1232 \mathrm{w}, 1141 \mathrm{~m}, 1074 \mathrm{~m}, 1017 \mathrm{~m}, 990 \mathrm{w}, 866 \mathrm{~m}, 755 \mathrm{~s}, 625 \mathrm{~m}$.

${ }^{1} \mathrm{H}$ NMR (500 MHz, $\mathrm{CDCl}_{3}$ ): $\delta=7.55$ (dd, ${ }^{3} \mathrm{~J} 7.9,{ }^{4} \mathrm{~J} 1.5,2 \mathrm{H}, \mathrm{C} 3 H$ ), 7.24 (ddd, ${ }^{3} J$ 8.0, 7.3, ${ }^{4} J 1.5,2 \mathrm{H}, \mathrm{C} 5 H$ ), 7.00 (dd, ${ }^{3} J$ 8.0, ${ }^{4} \mathrm{~J} 1.6,2 \mathrm{H}, \mathrm{C} 6 \mathrm{H}$ ), 6.94 (ddd, ${ }^{3}$ ) $\left.7.9,7.4,{ }^{4} \mathrm{~J} 1.6,2 \mathrm{H}, \mathrm{C} 4 \mathrm{H}\right), 3.23\left(\mathrm{~s}, 3 \mathrm{H}, \mathrm{NCH}_{3}\right)$.

${ }^{13} \mathrm{C}$ NMR (125 MHz, $\mathrm{CDCl}_{3}$ ): $\delta=148.7$ (C1), $134.4(C 3), 128.1(C 5), 124.8$ (C4), $123.8(\mathrm{C} 6), 120.3(\mathrm{C} 2), 41.3\left(\mathrm{CH}_{3}\right)$.

HRMS (ESI): $\mathrm{m} / \mathrm{z}$ calcd. for $\mathrm{C}_{13} \mathrm{H}_{11} \mathrm{Br}_{2} \mathrm{~N}^{+} 339.9331$ found $339.9334\left[\mathrm{M}+\mathrm{H}^{+}\right]$.

1,5-Bifunctional Organomagnesium Reagent for the Ester to Acridinium Transformation

To a suspension of magnesium turnings ( $13.6 \mathrm{mg}, 560 \mu \mathrm{mol})$ in anhydrous THF $(0.20 \mathrm{~mL})$ at $60{ }^{\circ} \mathrm{C}$ was added a solution of 2-bromo- $N-(2$ bromophenyl)- $N$-methylaniline (47.7 $\mathrm{mg}, 140 \mu \mathrm{mol}$ ) in anhydrous THF 
$(0.60 \mathrm{~mL})$. The mixture was stirred at $60^{\circ} \mathrm{C}$ for $3 \mathrm{~h}$ during which the reaction mixture turned yellow, leading to the 1,5-bifunctional organomagnesium reagent which was used directly in the next step.

\section{General Procedure A}

To a solution of the above reagent in THF $(140 \mu \mathrm{mol})$ at $60^{\circ} \mathrm{C}$ was added a solution of carboxylic acid ester $(100 \mu \mathrm{mol})$ in anhydrous THF $(1.00 \mathrm{~mL})$ and the reaction mixture was stirred at the same temperature for $12 \mathrm{~h}$. Aqueous $\mathrm{HBr}\left(1.00 \mathrm{~mL}, 8.8 \mathrm{molL}^{-1}\right)$ was added and the solvent was removed in vacuo. The residue was dissolved in $\mathrm{MeOH}$ and filtered over a bed of Amberlyst A21 free base. The solvent was removed in vacuo, the residue was dissolved in $\mathrm{CH}_{2} \mathrm{Cl}_{2}$ and $3.0 \mathrm{~g}$ of silica gel was added. The solvent was removed in vacuo and the residue purified by column chromatography with $\mathrm{CH}_{2} \mathrm{Cl}_{2} 100 \%$ to $\mathrm{CH}_{2} \mathrm{Cl}_{2}: \mathrm{MeOH} 100: 2$ to $100: 5$ to 100:8 to yield acridinium salts $\mathbf{1 a}$ and $\mathbf{1 b}$.

\section{9-(4-Fluorophenyl)-10-methylacridinium bromide salt (1a)}

Prepared according to general procedure A using methyl 4fluorobenzoate $(15.4 \mathrm{mg}, 100 \mu \mathrm{mol})$ and the 1,5-bifunctional organomagnesium reagent to afford a yellow solid (22.8 mg, $63 \%$, m.p. 212-214 $\left.{ }^{\circ} \mathrm{C}\right): \mathrm{R}_{f} 0.10\left(\mathrm{CH}_{2} \mathrm{Cl}_{2}: \mathrm{MeOH} 10: 1\right)$.

IR (ATR, neat): 3377w, 3059w, 2999w, 2923w, 2848w, 1601m, 1574m, $1544,1504 \mathrm{~m}, 1482 \mathrm{w}, 1446 \mathrm{w}, 1373 \mathrm{~m}, 1272 \mathrm{w}, 1217 \mathrm{~m}, 1156 \mathrm{~m}, 1094 \mathrm{~m}$, $1023 \mathrm{~m}, 926 \mathrm{w}, 866 \mathrm{w}, 816 \mathrm{w}, 767 \mathrm{~s}, 722 \mathrm{~m}, 666 \mathrm{~m}, 614 \mathrm{~m}$.

${ }^{1} \mathrm{H}$ NMR $\left(500 \mathrm{MHz}, \mathrm{CDCl}_{3}\right): \delta=9.05\left(\mathrm{~d},{ }^{3} J 9.2,2 \mathrm{H}, \mathrm{C} 4 H, \mathrm{C} 5 H\right), 8.41-8.44(\mathrm{~m}$, 2H, C3H, C6H), 7.97 (dd, ${ }^{3} J$ 8.8, ${ }^{4} \mathrm{~J} 1.2,2 \mathrm{H}, \mathrm{C} 1 H, \mathrm{C} 8 H$ ), 7.82 (dd, ${ }^{3} \mathrm{~J} 8.7,6.7$, $2 \mathrm{H}, \mathrm{C} 2 H, \mathrm{C} 7 H), 7.47-7.50\left(\mathrm{~m}, 2 \mathrm{H}, \mathrm{C}^{\prime} H, \mathrm{C}^{\prime} H\right), 7.42-7.45\left(\mathrm{~m}, 2 \mathrm{H}, \mathrm{C} 3^{\prime} H\right.$, $\left.\mathrm{C}^{\prime} H\right), 5.34\left(\mathrm{~s}, 3 \mathrm{H}, \mathrm{NCH}_{3}\right)$.

${ }^{13} \mathrm{C}$ NMR (125 MHz, $\mathrm{CDCl}_{3}$ ): $\delta=164.1$ (d, $\left.{ }^{1} J_{\mathrm{CF}} 253, C 4^{\prime}\right), 160.0$ (C9), 141.8 (C4a, C10a), $139.4(C 3, C 6), 132.0\left(\mathrm{~d},{ }^{3} J_{\mathrm{CF}} 8.3, C 2^{\prime}, C 6^{\prime}\right), 129.8(C 1, C 8), 128.8$ (d, $\left.{ }^{4} J_{\mathrm{CF}} 3.7, C 1^{\prime}\right) 128.3(C 2, C 7), 126.3(C 8 \mathrm{a}, C 9 \mathrm{a}), 120.0(C 4, C 5), 116.7$ (d, $\left.{ }^{2} J_{\mathrm{CF}} 22, C 3^{\prime}, \mathrm{C5}^{\prime}\right), 41.5\left(\mathrm{NCH}_{3}\right)$.

HRMS (ESI): $\mathrm{m} / \mathrm{z}$ calcd. for $\mathrm{C}_{20} \mathrm{H}_{15} \mathrm{FN}^{+} 288.1183$ found 288.1185 [M+].

Absorption spectroscopy (in $\mathrm{MeCN}$ ): $\lambda_{\text {abs }} 426 \mathrm{~nm}$; $\varepsilon_{\text {abs: }}$ 8.4.102 $\mathrm{Lcm}^{-1} \mathrm{~mol}^{-1}$; $\lambda_{\mathrm{em}}(\operatorname{exc} 410): 512 \mathrm{~nm}$; Stokes shift: $86 \mathrm{~nm}$; $\mathrm{E}_{0,0}: 2.83 \mathrm{eV} ;$ Cyclic voltammetry (vs $\mathrm{SCE}$ ): $\mathrm{E}_{1 / 2}\left(\mathrm{P}^{*} / \mathrm{P}^{-}\right):+2.32 \mathrm{~V}$, $\mathrm{E}_{1 / 2}\left(\mathrm{P} / \mathrm{P}^{-}\right):-0.51 \mathrm{~V}$.

\section{9-(4-Methoxyphenyl)-10-methylacridinium bromide salt (1b)}

Prepared according to general procedure A using 4-methoxybenzoate (16.6 mg, $100 \mu \mathrm{mol})$ and the 1,5-bifunctional organomagnesium reagent to afford an orange solid (29.6 mg, $78 \%$, decomp. at $185{ }^{\circ} \mathrm{C}$ ): $\mathrm{R}_{f} 0.16$ $\left(\mathrm{CH}_{2} \mathrm{Cl}_{2}: \mathrm{MeOH} 10: 1\right)$.

IR (ATR, neat): 3336m, 3092w, 2931w, 1605s, 1547m, 1457m, 1376m, 1250s, 1176s, 1113w, 1022m, 922w, 932m, 761s, 725s, 666m, 617s.

${ }^{1} \mathrm{H}$ NMR $\left(500 \mathrm{MHz}, \mathrm{CDCl}_{3}\right): \delta=8.93\left(\mathrm{~d},{ }^{3} \mathrm{~J} 8.8 \mathrm{~Hz}, 2 \mathrm{H}, \mathrm{C} 4 \mathrm{H}, \mathrm{C} 5 H\right), 8.34\left(\mathrm{t},{ }^{3} \mathrm{~J}\right.$ $7.5 \mathrm{~Hz}, 2 \mathrm{H}, \mathrm{C} 3 H, \mathrm{C} 6 H$ ), 8.02 (d, ${ }^{3} \mathrm{~J} 8.6,2 \mathrm{H}, \mathrm{C} 1 H, \mathrm{C} 8 H$ ), 7.73 (dd, ${ }^{3} J$ 8.9, 6.6, $2 \mathrm{H}, \mathrm{C} 2 \mathrm{H}, \mathrm{C} 7 \mathrm{H}), 7.33-7.37\left(\mathrm{~m}, 2 \mathrm{H}, \mathrm{C}^{\prime} H, \mathrm{C}^{\prime} H\right), 7.16-7.18\left(\mathrm{~m}, 2 \mathrm{H}, \mathrm{C} 3^{\prime} H\right.$, $\left.\mathrm{C}^{\prime} H\right), 5.23\left(\mathrm{~s}, 3 \mathrm{H}, \mathrm{NCH}_{3}\right), 3.93\left(3 \mathrm{H}, \mathrm{s}, \mathrm{OCH}_{3}\right)$.

${ }^{13} \mathrm{C}$ NMR (125 MHz, CDCl 3 ): $\delta=161.4\left(C 4^{\prime}\right), 161.4(C 9), 141.6$ (C4a, $\left.C 10 \mathrm{a}\right)$, $139.0(C 3, C 6), 131.7\left(C 2^{\prime}, C 6^{\prime}\right), 130.2(C 1, C 8), 127.8(C 2, C 7), 126.2$ (C8a, $C 9 \mathrm{a}), 124.8\left(C 1^{\prime}\right), 119.7(C 4, C 5), 114.6\left(C 3^{\prime}, C 5^{\prime}\right), 55.7\left(\mathrm{OCH}_{3}\right), 41.0\left(\mathrm{~N} C H_{3}\right)$. HRMS (ESI): $\mathrm{m} / \mathrm{z}$ calcd. for $\mathrm{C}_{21} \mathrm{H}_{18} \mathrm{NO}^{+} 300.1383$ found $300.1384\left[\mathrm{M}^{+}\right]$.

Absorption spectroscopy (in $\mathrm{MeCN}$ ): $\lambda_{\text {abs }} 438 \mathrm{~nm}$; Eabs: $3.2 \cdot 10^{2} \mathrm{Lcm}^{-1} \mathrm{~mol}^{-1}$; $\lambda_{\mathrm{em}}(\operatorname{exc} 360): 499 \mathrm{~nm}$; Stokes shift: $61 \mathrm{~nm}$; $\mathrm{E}_{0,0}: 2.77 \mathrm{eV}$; Cyclic voltammetry (vs SCE): $\mathrm{E}_{1 / 2}\left(\mathrm{P}^{*} / \mathrm{P}^{-}\right):+2.21 \mathrm{~V}$, $\mathrm{E}_{1 / 2}\left(\mathrm{P} / \mathrm{P}^{-}\right):-0.56 \mathrm{~V}$.

\section{Gram-Scale Synthesis of Acridinium Salt 1c and 1d}

4-Bromo- $\boldsymbol{N}^{1}, N^{1}$-dimethylbenzene-1,3-diamine (3)

[CAS Reg. No.: 90555-68-3]

Prepared according to a modified literature procedure. ${ }^{10 a}$ To a solution of 4-bromo- $N, N$-dimethylaniline $(5,50.0 \mathrm{~g}, 250 \mathrm{mmol})$ in TBME/THF (1: 1 , $340 \mathrm{~mL}$ ) was added dropwise aq. nitric acid $(68 \%, 16.5 \mathrm{~mL})$. The mixture was stirred for $1 \mathrm{~h}$ at room temperature. The formed precipitate was filtered off and the residue was washed with TBME $(2 \times 30 \mathrm{~mL})$ and dried under vacuum overnight. ${ }^{14}$ The yellow salt ( $\left.35.0 \mathrm{~g}, 133 \mathrm{mmol}\right)$ was then dissolved in $\mathrm{CH}_{2} \mathrm{Cl}_{2}(210 \mathrm{~mL})$ and the solution was added to conc. $\mathrm{H}_{2} \mathrm{SO}_{4}$ $(58.0 \mathrm{~mL})$ while maintaining the temperature below $5^{\circ} \mathrm{C}$. The mixture was allowed to warm up to room temperature and was stirred for $1 \mathrm{~h}$. The mixture was then slowly added to cold water $(210 \mathrm{~mL})$ and aq. $\mathrm{NH}_{4} \mathrm{OH}$ (28\%) was added until the mixture reached $\mathrm{pH} 10$. The aq. residue was extracted with DCM $(2 \times 500 \mathrm{~mL})$, the combined organic layers were dried over $\mathrm{Na}_{2} \mathrm{SO}_{4}$ and concentrated in vacuo to obtain 4-bromo- $N, \mathrm{~N}$-dimethyl3-nitroaniline as an orange solid (32.0 g, 52\%, m.p. 90.4-91.9 $\left.{ }^{\circ} \mathrm{C}\right) . \mathrm{R}_{f} 0.73$ $\left(\mathrm{CH}_{2} \mathrm{Cl}_{2} 100 \%\right)$.

IR (ATR, neat): 2920w, 1609m, 1530s, 1443w, 1369m, 1234w, 1190w, $1129 \mathrm{w}, 1069 \mathrm{w}, 881 \mathrm{w}, 679 \mathrm{w}$.

${ }^{1} \mathrm{H} \mathrm{NMR}\left(500 \mathrm{MHz}, \mathrm{CDCl}_{3}\right): \delta=7.46\left(\mathrm{~d},{ }^{3} \mathrm{~J} 9.0,1 \mathrm{H}, \mathrm{C} 5 H\right), 7.07\left(\mathrm{~d},{ }^{4} J 3.1,1 \mathrm{H}\right.$, $\mathrm{C} 2 H), 6.69\left(\mathrm{dd},{ }^{3} J 9.1,{ }^{4} J 3.1,1 \mathrm{H}, \mathrm{C} 6 H\right), 3.00\left(\mathrm{~s}, 6 \mathrm{H}, \mathrm{N}\left(\mathrm{CH}_{3}\right)_{2}\right)$.

${ }^{13} \mathrm{C}$ NMR (125 MHz, $\mathrm{CDCl}_{3}$ ): $\delta=150.4$ (C3), 149.7 (C1), 134.9 (C5), 116.4 (C6), $108.3(\mathrm{C} 2), 98.6(\mathrm{C} 4), 40.3\left(\mathrm{~N}\left(\mathrm{CH}_{3}\right)_{2}\right)$.

HRMS (ESI): $\mathrm{m} / \mathrm{z}$ calcd. for $\mathrm{C}_{8} \mathrm{H}_{10} \mathrm{BrN}_{2} \mathrm{O}_{2}+244.9920$ found 244.9921 $\left[\mathrm{M}+\mathrm{H}^{+}\right]$.

In agreement with literature data. ${ }^{3 a}$

Prepared according to a modified literature procedure. ${ }^{10 \mathrm{~b}}$ To a solution of 4-bromo- $N, N$-dimethyl-3-nitroaniline (36.8 g, $150 \mathrm{mmol})$ in methanol/ water $(1: 1,430 \mathrm{~mL})$, was added ammonium chloride ( $64.2 \mathrm{~g}, 1.20 \mathrm{~mol}$ ) and iron powder ( $41.9 \mathrm{~g}, 750 \mathrm{mmol})$. The reaction mixture was stirred for $1 \mathrm{~h}$ at $70^{\circ} \mathrm{C}$, filtered over celite and concentrated to remove methanol. ${ }^{15}$ The aq. layer was treated with aq. $\mathrm{HCl}(10 \%)$ and washed with diethylether ( $2 \times 500 \mathrm{~mL}) . \mathrm{NH}_{4} \mathrm{OH}$ aq. (28\%) was added until basic $\mathrm{pH}$ was reached. The aq. layer was extracted with $\mathrm{CH}_{2} \mathrm{Cl}_{2}(3 \times 1 \mathrm{~L})$. The combined organic layers were washed with brine, dried over $\mathrm{Na}_{2} \mathrm{SO}_{4}$ and concentrated in vacuo to yield 4-bromo- $N^{1}, N^{1}$-dimethylbenzene-1,3diamine as a black solid (28.1 g, $87 \%$, m.p. $\left.57.1-58.7^{\circ} \mathrm{C}\right) . \mathrm{R}_{f} 0.29\left(\mathrm{CH}_{2} \mathrm{Cl}_{2}\right.$ $100 \%)$.

IR (ATR, neat): 3460w, 3355w, 2956w, 2843w, 2794w, 1604s, 1568s, $1491 \mathrm{~m}, 1350 \mathrm{~m}, 1291 \mathrm{~m}, 1153 \mathrm{~m}, 1123 \mathrm{~m}, 1057 \mathrm{w}, 972 \mathrm{w}, 893 \mathrm{w}, 817 \mathrm{~s}, 778 \mathrm{~s}$, 710w.

${ }^{1} \mathrm{H}$ NMR (500 MHz, $\left.\mathrm{CDCl}_{3}\right): \delta=7.20\left(\mathrm{~d},{ }^{3} J 8.8,1 \mathrm{H}, \mathrm{C} 5 \mathrm{H}\right), 6.12\left(\mathrm{~d},{ }^{4} J 2.9,1 \mathrm{H}\right.$, $\mathrm{C} 2 \mathrm{H}$ ) $6.08\left(\mathrm{dd}, 3 \mathrm{~J} 8.8,{ }^{4} \mathrm{~J} 2.9,1 \mathrm{H}, \mathrm{C} 6 \mathrm{H}\right), 3.97(\mathrm{br}, 2 \mathrm{H}, \mathrm{NH} 2$ ), $2.88(\mathrm{~s}, 6 \mathrm{H}$, $\left.\mathrm{N}\left(\mathrm{CH}_{3}\right)_{2}\right)$.

${ }^{13} \mathrm{C}$ NMR (125 MHz, $\mathrm{CDCl}_{3}$ ): $\delta=151.0(C 1), 144.3$ (C3), 132.5 (C5), 105.2 (C6), $99.7(\mathrm{C} 2), 97.0(\mathrm{C} 4), 40.6\left(\mathrm{~N}\left(\mathrm{CH}_{3}\right) 2\right)$.

HRMS (ESI): m/z calcd. for $\mathrm{C}_{8} \mathrm{H}_{12} \mathrm{BrN}_{2}{ }^{+} 215.0178$ found $215.0181\left[\mathrm{M}+\mathrm{H}^{+}\right]$. In agreement with literature data. ${ }^{3 a}$

\section{4-Bromo-3-iodo- $\mathrm{N}, \mathrm{N}$-dimethylaniline (4)}

[CAS Reg. No.: 1291063-32-5]

Prepared according to a modified literature procedure. ${ }^{9 a}$ To a suspension of NaI $(39.0 \mathrm{~g}, 260 \mathrm{mmol}), \quad \mathrm{CuI}(1.24 \mathrm{~g}, 6.50 \mathrm{mmol}), N, N^{\prime}-$ dimethylethylenediamine $(1.40 \mathrm{~mL}, 13.0 \mathrm{mmol})$ in 1,4 -dioxane $(130 \mathrm{~mL})$ at RT was added 3-bromo- $N, N$-dimethylaniline $(18.6 \mathrm{~mL}, 130 \mathrm{mmol})$. The reaction mixture was stirred for $22 \mathrm{~h}$ at $110{ }^{\circ} \mathrm{C}$ and then cooled to RT, treated with aq. $\mathrm{NH}_{4} \mathrm{OH}(28 \%, 650 \mathrm{~mL})$ and $\mathrm{H}_{2} \mathrm{O}(300 \mathrm{~mL})$ and was extracted with $\mathrm{CH}_{2} \mathrm{Cl}_{2}(3 \times 1.5 \mathrm{~L})$. The combined organic layers were dried over $\mathrm{Na}_{2} \mathrm{SO}_{4}$ and concentrated in vacuo to give 3-iodo- $N, N$ dimethylaniline as a brownish oil (31.3 g, 97\%). 
${ }^{1} \mathrm{H}$ NMR (500 MHz, $\left.\mathrm{CDCl}_{3}\right): \delta=7.01-7.03(\mathrm{~m}, 2 \mathrm{H}, \mathrm{C} 4 H, \mathrm{C} 6 H), 6.91-6.94(\mathrm{~m}$, $1 \mathrm{H}, \mathrm{C} 5 \mathrm{H}), 6.65-6.67(\mathrm{~m}, 1 \mathrm{H}, \mathrm{C} 2 \mathrm{H}), 2.92\left(\mathrm{~s}, 6 \mathrm{H}, \mathrm{N}\left(\mathrm{CH}_{3}\right)_{2}\right)$.

${ }^{13} \mathrm{C}$ NMR (125 MHz, $\left.\mathrm{CDCl}_{3}\right): \delta=151.6$ (C1), 130.4 (C5), 125.2 (C4), 121.1 (C6), $111.6(\mathrm{C} 2), 95.5(\mathrm{C} 3), 40.3\left(\mathrm{~N}\left(\mathrm{CH}_{3}\right)_{2}\right)$.

In agreement with literature data. ${ }^{\mathrm{b}}$

To a solution of 3-iodo- $\mathrm{N}, \mathrm{N}$-dimethylaniline (31.3 g, $127 \mathrm{mmol}$ ) in $\mathrm{CH}_{2} \mathrm{Cl}_{2}$ $(200 \mathrm{~mL})$ at $10{ }^{\circ} \mathrm{C}$ was added bromine $(6.53 \mathrm{~mL}, 127 \mathrm{mmol})$. The reaction mixture was stirred $1 \mathrm{~h}$ at that temperature. ${ }^{16}$ The suspension was treated with aq. sat. $\mathrm{Na}_{2} \mathrm{SO}_{3}(76.0 \mathrm{~mL})$ and $\mathrm{H}_{2} \mathrm{O}(170 \mathrm{~mL})$ and was extracted with $\mathrm{CH}_{2} \mathrm{Cl}_{2}(3 \times 350 \mathrm{~mL})$. The combined organic layers were dried over $\mathrm{Na}_{2} \mathrm{SO}_{4}$ and concentrated in vacuo. The red crude solid was recrystallized from hexane/EtOAc (50 mL; 4:1) to give 4-bromo-3-iodo- $N, N$-dimethylaniline as a beige solid ( $23.7 \mathrm{~g}, 57 \%) .{ }^{17}$

In agreement with literature data. ${ }^{9 c}$

\section{4-Bromo- $N^{3}$-(2-bromo-5-(dimethylamino)phenyl)- $N^{1}, N^{1}, N^{3}$ - trimethylbenzene-1,3-diamine (2b)}

To a degassed mixture of 4-bromo-3-iodo- $N, N$-dimethylaniline $[55.4 \mathrm{~g}$, $170 \mathrm{mmol}$ ), 4-bromo- $N^{1}, N^{1}$-dimethylbenzene-1,3-diamine (36.6 g, $170 \mathrm{mmol}$ ) tris(dibenzylideneacetone)dipalladium (3.89 g, $4.25 \mathrm{mmol}$ ), 1,1'-bis(diphenylphosphino)ferrocene $(4.71 \mathrm{~g}, 8.50 \mathrm{mmol})$ and sodium $t$ butoxide $(24.5 \mathrm{~g}, 255.0 \mathrm{mmol})$ was added toluene $(560 \mathrm{~mL})$ at RT. The reaction mixture was stirred for $14 \mathrm{~h}$ at $105^{\circ} \mathrm{C}$. The solution was diluted with $\mathrm{H}_{2} \mathrm{O}(800 \mathrm{~mL})$ and extracted with $\mathrm{CH}_{2} \mathrm{Cl}_{2}(3 \times 2 \mathrm{~L})$. The combined organic layer was dried over $\mathrm{Na}_{2} \mathrm{SO}_{4}$ and concentrated in vacuo. The crude was purified by column chromatography on silica gel (pentane $/ \mathrm{CH}_{2} \mathrm{Cl}_{2} 3: 1$ to $2: 1$ to $1: 1)$ giving 4 -bromo- $N^{3}$-(2-bromo-5-(dimethylamino)phenyl)$N^{1}, N^{1}$-dimethyl-benzene-1,3-diamine as a beige solid (48.9 g, 70\%, m.p. $\left.132.2-134.7^{\circ} \mathrm{C}\right) . \mathrm{R}_{f} 0.53 \mathrm{CH}_{2} \mathrm{Cl}_{2}(100 \%)$.

IR (ATR, neat): 3399w, 2895w, 2804w, 1590m, 1562s, 1496s, $1441 \mathrm{~m}$, 1354s, 1284s, 1230w, 1161m, 1065w, 989w, 921w, 814m, 770s, 737w, $682 \mathrm{w}$.

${ }^{1} \mathrm{H}$ NMR $\left(500 \mathrm{MHz}, \mathrm{CDCl}_{3}\right): \delta=7.36\left(\mathrm{~d},{ }^{3} J 8.7,2 \mathrm{H}, \mathrm{C} 3 H\right), 6.76\left(\mathrm{~d},{ }^{4} \mathrm{~J} 2.9,2 \mathrm{H}\right.$, $\mathrm{C} 6 H$ ) $6.32(\mathrm{br}, 1 \mathrm{H}, \mathrm{NH}), 6.24$ (dd, $\left.{ }^{3} \mathrm{~J} \mathrm{8.7,}{ }^{4} \mathrm{~J} 3.0,2 \mathrm{H}, \mathrm{C} 4 H\right) 2.89(\mathrm{~s}, 12 \mathrm{H}$, $\left.\mathrm{N}\left(\mathrm{CH}_{3}\right)_{2}\right)$.

${ }^{13} \mathrm{C}$ NMR (125 MHz, $\mathrm{CDCl}_{3}$ ): $\delta=150.5$ (C5), 140.3 (C1), $133.0(C 3), 107.3$ (C4), 102.1 (C6), $100.8(C 2), 40.7\left(\mathrm{~N}\left(\mathrm{CH}_{3}\right)_{2}\right)$.

HRMS (ESI): $\mathrm{m} / \mathrm{z}$ calcd. for $\mathrm{C}_{16} \mathrm{H}_{20} \mathrm{Br}_{2} \mathrm{~N}_{3}{ }^{+} 412.0018$ found 412.0019 $\left[\mathrm{M}+\mathrm{H}^{+}\right]$.

Prepared according to a modified literature procedure. ${ }^{7}$ To a solution of of 4-bromo- $N^{3}$-(2-bromo-5-(dimethylamino) phenyl)- $N, N^{1}$-dimethylbenzene-1,3-diamine ( $48.3 \mathrm{~g}, 117.0 \mathrm{mmol}$ ) in THF (334 $\mathrm{mL})$ at RT was added sodium hydride $(60 \%$ dispersion in mineral oil, $14.0 \mathrm{~g}, 351.0$ mmol). The suspension was heated to $75^{\circ} \mathrm{C}$ and stirred for $30 \mathrm{~min}$ at this temperature. Iodomethane $(7.28 \mathrm{~mL}, 117.0 \mathrm{mmol})$ was added within 5 min at $75{ }^{\circ} \mathrm{C}$ and the reaction mixture was stirred for $2 \mathrm{~h}$ at this temperature. The suspension was treated with water $(585 \mathrm{~mL})$ and extracted with $\mathrm{CH}_{2} \mathrm{Cl}_{2}(3 \times 1.5 \mathrm{~L})$. The combined organic layers were dried over $\mathrm{Na}_{2} \mathrm{SO}_{4}$ and concentrated in vacuo. The crude solid was recrystallized from hexane/EtOAc $(70 \mathrm{~mL} ; 4: 1)$ to give 4-bromo- $N^{3}$-(2-bromo-5(dimethylamino)phenyl)- $N^{1}, N^{1}, N^{3}$-trimethylbenzene-1,3-diamine (2b) as a beige solid (35.6 g, 71\%, m.p. $\left.102.5-104.1^{\circ} \mathrm{C}\right): \mathrm{R}_{f} 0.55\left(\mathrm{CH}_{2} \mathrm{Cl}_{2} 100 \%\right)$.

IR (ATR, neat): 2883w, 2806w, 1588s, 1554s, 1492s, 1446m, 1358s, $1304 \mathrm{~m}, 1233 \mathrm{~m}, 1170 \mathrm{~s}, 1133 \mathrm{~m}, 1084 \mathrm{~m}, 1022 \mathrm{w}, 987 \mathrm{~m}, 909 \mathrm{w}, 801 \mathrm{~m}$, $735 \mathrm{w}$.

${ }^{1} \mathrm{H}$ NMR $\left(500 \mathrm{MHz}, \mathrm{CDCl}_{3}\right): \delta=7.33\left(\mathrm{~d},{ }^{3} J 8.8,2 \mathrm{H}, \mathrm{C} 3 H\right) ; 6.35\left(\mathrm{~d},{ }^{4} J 3.0,2 \mathrm{H}\right.$, $\mathrm{C} 6 H), 6.31\left(\mathrm{dd},{ }^{3} \mathrm{~J} 8.8,{ }^{4} J 3.0,2 \mathrm{H}, \mathrm{C} 4 H\right), 3.21\left(\mathrm{~s}, 3 \mathrm{H}, \mathrm{NCH}_{3}\right), 2.87(\mathrm{~s}, 12 \mathrm{H}, 2 \mathrm{x}$ $\left.\mathrm{N}\left(\mathrm{CH}_{3}\right)_{2}\right)$.

${ }^{13} \mathrm{C}$ NMR (125 MHz, $\mathrm{CDCl}_{3}$ ): $\delta=150.7$ (C5), $149.1(C 1), 134.0(C 3), 109.1$ (C4), 108.3 (C6), $106.7(\mathrm{C} 2), 41.3\left(\mathrm{NCH}_{3}\right), 40.6\left(2 \times \mathrm{N}\left(\mathrm{CH}_{3}\right)_{2}\right)$.

HRMS (ESI): $\mathrm{m} / \mathrm{z}$ calcd. for $\mathrm{C}_{17} \mathrm{H}_{22} \mathrm{Br}_{2} \mathrm{~N}_{3}+426.0175$ found 426.0177 $\left[\mathrm{M}+\mathrm{H}^{+}\right]$.

\section{General Procedure B}

To a suspension of magnesium turnings ( $1.36 \mathrm{~g}, 56.0 \mathrm{mmol})$ in anhydrous THF $(20.0 \mathrm{~mL})$ at $60^{\circ} \mathrm{C}$ was added a solution of 4-bromo- $N^{3}$-(2-bromo-5(dimethylamino)phenyl)- $N^{1}, N^{1}, N^{3}$-trimethylbenzene-1,3-diamine (2b, $5.98 \mathrm{~g}, 14.0 \mathrm{mmol})$ in anhydrous THF $(60 \mathrm{~mL})$ followed by 1,2 dibromoethane $(0.319 \mathrm{~mL}, 4.20 \mathrm{mmol})$. The mixture was stirred at $60{ }^{\circ} \mathrm{C}$ for $3 \mathrm{~h}$ during which the reaction mixture turned yellow. To this solution at $60{ }^{\circ} \mathrm{C}$ was added a solution of carboxylic acid ester $(10.0 \mathrm{mmol})$ in anhydrous THF $(100 \mathrm{~mL})$ and the reaction mixture was stirred at the same temperature for $12 \mathrm{~h}$. Aqueous $\mathrm{HBr}(100 \mathrm{~mL}, 8.8 \mathrm{molL}-1)$ was added and the aq. phase extracted with $\mathrm{CHCl}_{3} / \mathrm{i}-\mathrm{PrOH}(3 \times 200 \mathrm{~mL} ; 85: 15)$. The solvent was removed in vacuo and the residue was recrystallized from water $(10 \mathrm{~mL})$ to yield acridinium salts $\mathbf{1 c}$ and $\mathbf{1 d}$.

\section{3,6-Bis(dimethylamino)-9-mesityl-10-methylacridinium bromide salt (1c)}

Prepared according to general procedure B using methyl 2,4,6 trimethyl benzoate ( $1.78 \mathrm{~g}, 10 \mathrm{mmol})$ to afford an orange solid (3.9 g, 82\%, decomp. at $\left.192.9^{\circ} \mathrm{C}\right): \mathrm{R}_{f} 0.13\left(\mathrm{CH}_{2} \mathrm{Cl}_{2}: \mathrm{MeOH} 10: 1\right)$.

IR (ATR, neat): 3373w, 2918w, 2170w, 1591s, 1497s, 1435m, 1352s, $1210 \mathrm{~s}, 1148 \mathrm{~s}, 985 \mathrm{w}, 921 \mathrm{~s}, 807 \mathrm{~m}, 711 \mathrm{~s}$.

${ }^{1} \mathrm{H} \mathrm{NMR}\left(500 \mathrm{MHz}, \mathrm{CDCl}_{3}\right): \delta=7.26\left(\mathrm{~d},{ }^{3} J 9.5,2 \mathrm{H}, \mathrm{C} 1 H, \mathrm{C} 8 H\right), 7.06(\mathrm{br}, 2 \mathrm{H}$, $\left.\mathrm{C}^{\prime} H, \mathrm{C}^{\prime} H\right), 6.95-6.96(\mathrm{~m}, 2 \mathrm{H}, \mathrm{C} 4 H, \mathrm{C} 5 H), 6.94\left(\mathrm{dd},{ }^{3} J 9.4,{ }^{4} \mathrm{~J} 2.1,2 \mathrm{H}, \mathrm{C} 2 \mathrm{H}\right.$, $\mathrm{C} 7 \mathrm{H}), 4.56\left(\mathrm{~s}, 3 \mathrm{H}, \mathrm{NCH}_{3}\right), 3.36\left(\mathrm{~s}, 12 \mathrm{H}, 2 \times \mathrm{x}\left(\mathrm{CH}_{3}\right)_{2}\right), 2.43\left(\mathrm{~s}, 3 \mathrm{H}, \mathrm{CH}_{3}\right) 1.80$ (s, 6H, $\left.2 \times \mathrm{CH}_{3}\right)$.

${ }^{13} \mathrm{C}$ NMR (125 MHz, $\left.\mathrm{CDCl}_{3}\right): \delta=155.4(C 3, C 6), 154.0(C 9), 143.9$ (C4a, C10a), 139.0 (C4'), 136.0 (C2',C6'), 130.4 (C1,C8,C1'), $128.6\left(C 3^{\prime}, C 5^{\prime}\right)$, 116.1 (C8a, $C 9 \mathrm{a}), 114.3(C 2, C 7), 94.3(C 4, C 5), 41.1\left(2 \times \mathrm{N}\left(\mathrm{CH}_{3}\right)_{2}\right), 38.3$ $\left(\mathrm{NCH}_{3}\right), 21.2\left(\mathrm{CH}_{3}\right), 19.8\left(2 \times \mathrm{CH}_{3}\right)$.

HRMS (ESI): m/z calcd. For $\mathrm{C}_{27} \mathrm{H}_{32} \mathrm{~N}_{3}+398.2591$ found $398.2594\left[\mathrm{M}+\mathrm{H}^{+}\right]$. In agreement with literature data. ${ }^{3 a}$

\section{3,6-Bis(dimethylamino)-9-(2,6-Dimethylphenyl)-10- methylacridinium bromide salt (1d)}

Prepared according to general procedure B using methyl 2,4,6 trimethyl benzoate $(1.78 \mathrm{~g}, 10 \mathrm{mmol})$ to afford an orange solid (3.9 g, 84\%, decomp. at $\left.238.6{ }^{\circ} \mathrm{C}\right): \mathrm{R}_{f} 0.17\left(\mathrm{CH}_{2} \mathrm{Cl}_{2}: \mathrm{MeOH} 10: 1\right)$.

IR (ATR, neat): 2917w, 2662w, 1595m, 1502m, 1439s, 1354m, 1259s, $1215 \mathrm{~m}, 1147 \mathrm{~m}, 1067 \mathrm{~s}, 981 \mathrm{~s}, 924 \mathrm{~m}, 809 \mathrm{~m}, 712 \mathrm{~s}, 649 \mathrm{~s}$.

${ }^{1} \mathrm{H}$ NMR (500 MHz, MeOD): $\delta=7.44\left(\mathrm{t},{ }^{3} J 7.6,1 \mathrm{H}, \mathrm{C}^{\prime} H\right), 7.31\left(\mathrm{~d},{ }^{3} J 7.6,2 \mathrm{H}\right.$, $\mathrm{C}^{\prime} H, \mathrm{C}^{\prime} H$ ) $7.28\left(\mathrm{~d}, 3^{3} \mathrm{9} 9.5,2 \mathrm{H}, \mathrm{C} 1 H, \mathrm{C} 8 H\right.$ ) $7.18\left(\mathrm{dd}, 3^{3} \mathrm{9} 9.5,{ }^{4} \mathrm{~J} 2.2,2 \mathrm{H}, \mathrm{C} 2 \mathrm{H}\right.$, $\mathrm{C} 7 \mathrm{H}), 6.88(\mathrm{~d}, 4 \mathrm{~J} 2.2,2 \mathrm{H}, \mathrm{C} 4 \mathrm{H}, \mathrm{C} 5 H), 4.30\left(\mathrm{~s}, 3 \mathrm{H}, \mathrm{NCH}_{3}\right), 3.34(\mathrm{~s}, 12 \mathrm{H}, 2 \mathrm{x}$ $\left.\mathrm{N}\left(\mathrm{CH}_{3}\right)_{2}\right), 1.85\left(\mathrm{~s}, 6 \mathrm{H}, 2 \times \mathrm{CH}_{3}\right)$.

${ }^{13} \mathrm{C}$ NMR (125 MHz, MeOD): $\delta=157.2$ (C3, C6), 155.3 (C9), 145.4 (C4a, C10a), 137.3 (C2', $\left.C 6^{\prime}\right), 135.0\left(C 1^{\prime}\right), 131.4(C 1, C 8), 130.6\left(C 4^{\prime}\right), 129.1$ (C3', $\left.C^{\prime}\right), 117.3$ (C8a, $\left.C 9 \mathrm{a}\right), 116.1(\mathrm{C} 2, C 7), 94.5(C 4, C 5), 40.8\left(2 \times \mathrm{N}\left(\mathrm{CH}_{3}\right)_{2}\right), 36.7$ $\left(\mathrm{NCH}_{3}\right), 19.8\left(2 \times \mathrm{CH}_{3}\right)$.

HRMS (ESI): $\mathrm{m} / \mathrm{z}$ calcd. For $\mathrm{C}_{26} \mathrm{H}_{30} \mathrm{~N}_{3}{ }^{+} 384.2434$ found $384.2440\left[\mathrm{M}+\mathrm{H}^{+}\right]$.

Absorption spectroscopy (in $\mathrm{MeCN}$ ): $\lambda_{\mathrm{abs}}$ : $504 \mathrm{~nm}$; $\varepsilon_{\text {abs: }}$ 7.1.10 $\mathrm{Lcm}^{-1} \mathrm{~mol}^{-1}$; $\lambda_{\mathrm{em}}(\operatorname{exc} 450)$ : $533 \mathrm{~nm}$; Stokes shift: $29 \mathrm{~nm}$; $\mathrm{E}_{0,0}: 2.4 \mathrm{eV}$; Cyclic voltammetry (vs $\left.\mathrm{SCE}\right): \mathrm{E}_{1 / 2}\left(\mathrm{P}^{*} / \mathrm{P}^{-}\right):+1.25 \mathrm{~V}$, $\mathrm{E}_{1 / 2}\left(\mathrm{P} / \mathrm{P}^{-}\right):-1.15 \mathrm{~V}$.

\section{Photoredox Catalyzed Deuteration}

\section{General Procedure C}

According to a modified literature procedure.11 To a mixture of triisopropylsilanethiol $(5.71 \mathrm{mg}, 30 \mu \mathrm{mol})$, lithium carbonate $(35.5 \mathrm{mg}$, $480 \mu \mathrm{mol})$, photocatalyst (1-2.5 mol\%, see table 3 ) and deuterium oxide (90.0 $\mu \mathrm{L}, 5.00 \mathrm{mmol})$ in $N$-methylpyrrolidone $(1.6 \mathrm{~mL})$ was added clomipramine hydrochloride (33.7 mg, $100 \mu \mathrm{mol}$ ) and was degassed by a stream of argon for $20 \mathrm{~min}$. The reaction was performed under an argon 
atmosphere for $24 \mathrm{~h}$ with the light on. The reaction mixture was diluted in EtOAc $(15 \mathrm{~mL})$, washed with brine $(20 \mathrm{~mL})$ and extracted with EtOAc ( $3 \times 15 \mathrm{~mL}$ ). The combined organic layer was concentrated in vacuo and the residue purified by column chromatography with $n$ hexane:acetone: $\mathrm{Et}_{3} \mathrm{~N}, 96: 2: 2$ to afford clomipramine free base. $\mathrm{R}_{f} 0.34(\mathrm{n}$ hexane:acetone: $\left.\mathrm{Et}_{3} \mathrm{~N}, 8: 1: 1\right)$.

${ }^{1} \mathrm{H}$ NMR (500 MHz, MeOD): $\delta=7.12-7.17(\mathrm{~m}, 3 \mathrm{H}), 7.09-7.10(\mathrm{~m}, 1 \mathrm{H})$, 7.02-7.03 (m, 1H), 6.95-6.98 (m, 1H), 6.85-6.87 (m, 1H), 3.73-3.76 (m, $\left.1.75 \mathrm{H}, 12 \% 2 \mathrm{H}, \mathrm{C}^{\prime} \mathrm{H}\right), 3.07-3.15(\mathrm{~m}, 4 \mathrm{H}, \mathrm{C} 5 \mathrm{H}, \mathrm{C} 6 \mathrm{H}), 2.33-2.40(\mathrm{~m}, 1.03 \mathrm{H}$, $\left.49 \% 2 \mathrm{H}, \mathrm{C} 3{ }^{\prime} \mathrm{H}\right), 2.11-2.15\left(\mathrm{~m}, 4.16 \mathrm{H}, 30 \% 2 \mathrm{H}, \mathrm{N}\left(\mathrm{CH}_{3}\right)_{2}\right), 1.70-1.74(\mathrm{~m}, 2 \mathrm{H}$, C2' $H$ ).

In agreement with literature. ${ }^{11}$

To afford the clomipramine $\mathrm{HCl}$ salt, the free base was dissolved in EtOAc, treated with $\mathrm{HCl}$ in dioxane $\left(4 \mathrm{molL}^{-1}, 250 \mu \mathrm{L}\right)$, concentrated in vacuo, triturated from $\mathrm{Et}_{2} \mathrm{O}$ and filtered.

\section{Acknowledgment}

We gratefully acknowledge the Swiss National Science Foundation, and the Department of Chemistry of the University of Basel for financial support. We thank Prof. O. S. Wenger for the use of equipment to acquiring photophysical data and Lukas Schneider for skillful experimental support. Compounds $\mathbf{7 a - f}$ are part of a filed patent (C. Fischer, C. Sparr. EP $17 / 188,288$ ) licensed to Solvias. The catalysts will be commercially available.

\section{References}

(1) (a) Ohkubo, K.; Mizushima, K; Iwata, R.; Souma, K.; Suzuki, N.; Fukuzumi, S. Chem. Commun. 2010, 46, 601. (b) H. Kotani, K. Ohkubo, S. Fukuzumi, J. Am. Chem. Soc. 2004, 126, 15999.

(2) (a) Romero, N. A.; Nicewicz, D. A. Chem. Rev. 2016, 116, 10075. (b) Brasholz M. in Science of Synthesis: Photocatalysis in Organic Synthesis, (Ed.: B. König), Thieme, Stuttgart, 2019, pp. 371-389.

(3) (a) Fischer, C.; Sparr, C. Angew. Chem. Int. Ed. 2018, 57, 2436. (b) Fischer, C.; Sparr, C. Synlett 2018, 29, 2176. (c) Fischer, C.; Sparr, C. Tetrahedron 2018, 74, 5486.

(4) (a) White, A. R.; Wang, L.; Nicewicz, D. A. Synlett. 2019, 30, 827. Other approaches: (b) Gini, A.; Uygur, M.; Rigotti, T.; Alemán, J.; García Mancheño, O. Chem. Eur. J. 2018, 24, 12509. (c) Bernthsen, A.; Bender, F. Ber. Dtsch. Chem. Ges. 1883, 16, 1802.

(5) Benniston, A. C.; Elliott, K. J.; Harrington, R. W.; Clegg, W. Eur. J. Org. Chem. 2009, 253.

(6) (a) Link, A.; Fischer, C.; Sparr, C. Angew. Chem. Int. Ed. 2015, 54, 1263. (b) Link, A.; Fischer, C.; Sparr, C. Synthesis. 2017, 49, 397. (c) Knochel, P.; Krasovskiy, A.; Sapountzis, I.; in Handbook of Functionalized Organometallics: Applications in Synthesis (Ed.: P. Knochel), Wiley-VCH, Weinheim, 2005.

(7) Uchiyama, M.; Matsumoto, Y.; Nakamura, S.; Ohwada, T.; Kobayashi, N.; Yamashita, N.; Matsumiya, A.; Sakamoto, T. J. Am. Chem. Soc. 2004, 126, 8755.

(8) Rzymski, T.; Zarebski, A.; Dreas, A.; Osowska, K.; Kucwaj, K.; Fogt, J.; Cholody, M.; Galezowski, M.; Czardybon, W.; Horvath, R.; Wiklik, K.; Milik, K.; Brózka, K. WO072435 2014.

(9) (a) Klapars, A.; Buchwald, S. L. J. Am. Chem. Soc. 2002, 124, 14844 (b) Jiang, X.; Wang, C.; Wei, Y.; Xue, D.; Liu, Z.; Xiao, J. Chem. Eur. J. 2014, 20, 58. (c) Kowada, T.; Yamaguchi, S.; Fujinaga, H.; Ohe, K. Tetrahedron 2011, 67, 3105.

(10) (a) Zhang, P.; Cedilote, M.; Cleary, T. P.; Pierce, M. E. Tetrahedron Lett. 2007, 48, 8659. (b) Hameed, P. S.; Patil, V.; Solapure, S.; Sjarma, U.; Madhavapeddi, P.; Raichurkar, A.; Chinnapattu, M.; Manjrekar, P.; Shanbhag, G.; Puttur, J.; Shinde, V.; Menasinakai, S.; Rudrapatana, S.; Achar, V.; Awasthy, D.; Nandishaiah, R.; Humnabadkar, V.; Ghosh, A.; Narayan, C.; Ramya, V. K.; Kaur, P.; Sharma, S.; J. Werngren, J.; Hoffner, S.; Panduga, V.; Kumar, C. N. N.; Reddy, J.; Kumar, M. K. N.; Ganguly, S.; Bharath, S.; Bheemarao, U. ; Mukherjee, K.; Arora, U.; Gaonkar, S.; Coulson, M.; Waterson, D.; Sambandamurthy, V. K.; de Sousa, S. M. J. Med. Chem. 2014, 57, 4889-4905.
(11) Loh, Y. Y.; Nagao, K.; Hoover, A. J.; Hesk, D.; Rivera, N. R.; Colletti, S. L.; Davies, I. W.; MacMillan, D. W. C. Science. 2017, 358, 1182.

(12) Fischer, C.; Kerzig, C.; Wenger, O. Sparr, C. Manuscript submitted.

(13) Xu, S.; Haeffner, F.; Li, B.; Zakharov, L. N.; Liu, S.-Y. Angew. Chem. Int. Ed. 2014, 53, 6795.

(14) The precipitate slowly formed after the addition of nitric acid and can be either grey or yellow (it will turn eventually yellow after drying under vacuum).

(15) The celite was washed with EtOAc.

(16) The reaction mixture turns beige immediately and the color remains until the end of the reaction.

(17) After heating up to $80^{\circ} \mathrm{C}$, a hot filtration allowed to remove insoluble red solids, while the precipitate of product could be isolated at RT. 